\title{
A tropical interpretation of $m$-dissimilarity maps
}

\author{
Cristiano Bocci * and Filip Cools ${ }^{\dagger}$
}

\begin{abstract}
Let $T$ be a weighted tree with $n$ numbered leaves and let $D=(D(i, j))_{i, j}$ be its distance matrix, so $D(i, j)$ is the distance between the leaves $i$ and $j$. If $m$ is an integer satisfying $2 \leq m \leq n$, we prove a tropical formula to compute the $m$-dissimilarity map of $T$ (i.e. the weights of the subtrees of $T$ with $m$ leaves), given $D$. For $m=3$, we present a tropical description of the set of $m$-dissimilarity maps of trees. For $m=4$, a partial result is given.
\end{abstract}

MSC. 05C05, 05C12, 14M15, 14Q99, 15A99, 92B05

\section{Introduction}

Let $D$ be a matrix whose rows and columns are indexed by a set $X$. We assume that $D$ is symmetric and has zero entries on the main diagonal. In phylogenetics, these kind of matrices are called dissimilarity matrices . Usually, we take $X=[n]:=\{1,2, \ldots, n\}$. Hence a dissimilarity matrix $D$ can also be seen as a map $D:[n]^{2} \rightarrow \mathbb{R}$, with $D(i, j)=D(j, i)$ and $D(i, i)=0$ for each $i, j \in[n]$.

A metric is a non-negative dissimilarity matrix which satisfies the triangle inequality $D(i, j) \leq D(i, k)+D(k, j)$ for all $i, j, k \in X$.

We say that $D$ has a graph realization if there is a weighted graph (so a nonnegative weight is assigned to each edge) whose node set contains $X$ and such that the distance (i.e. the length of the shortest path) between nodes $i, j \in X$ is exactly $D(i, j)$. A distance matrix is a non-negative dissimilarity matrix that has a graph realization. In [3, 4, one can find some results on these kind of matrices.

In the case the graph is a tree and $X$ corresponds to the set of leaves, $D$ is called a tree metric. This case has been studied intensively and is well understood. The main result is the following (see [2] or [6, Theorem 2.36]).

Theorem 1.1 (Tree Metric Theorem). Let $D$ be a non-negative dissimilarity matrix on $[n]$. Then $D$ is a tree metric on $[n]$ if and only if, for every four (not necessarily distinct) elements $i, j, k, l \in[n]$, the maximum of the three numbers

*I.T.I.S. "A. Avogadro", Via case Nuove 27, 53021 Abbadia San Salvatore (SI), Italy, email: cristiano.bocci@gmail.com .

${ }^{\dagger}$ K.U.Leuven, Department of Mathematics, Celestijnenlaan 200B, B-3001 Leuven, Belgium, email: Filip.Cools@wis.kuleuven.be . 
$D(i, j)+D(k, l), D(i, k)+D(j, l)$ and $D(i, l)+D(j, k)$ is attained at least twice. Moreover, the tree $T$ with leaves $[n]$ that realizes $D$ is unique.

The condition of the theorem is called the four-point condition. It is a necessary and sufficient condition on a matrix to be realized by a tree.

Tree metrics on $n$ leaves are parameterized by the space of trees $\mathcal{T}_{n} \subset \mathbb{R}^{\left(\begin{array}{l}n \\ 2\end{array}\right)}$. The following result gives us a description of $\mathcal{T}_{n}$ (see [1]).

Theorem 1.2. The space of trees $\mathcal{T}_{n}$ is the union of $(2 n-5) ! !=1.3 .5 \ldots(2 n-5)$ orthants isomorphic to $\mathbb{R}_{\geq 0}^{2 n-3}$. More precisely, $\mathcal{T}_{n}$ is a simplicial fan of pure dimension $2 n-3$ in $\mathbb{R}^{\left(\begin{array}{c}n \\ 2\end{array}\right)}$.

We can consider a generalisation of the concept of dissimilarity matrix. Let $m \leq n$ be an integer. A map $D:[n]^{m} \rightarrow \mathbb{R}$ is called an $m$-dissimilarity map if

$$
D\left(i_{1}, \ldots, i_{m}\right)=D\left(i_{\pi(1)}, \ldots, i_{\pi(m)}\right)
$$

for all permutations $\pi \in S_{m}$ and $D\left(i_{1}, i_{2}, \ldots, i_{m}\right)=0$ if the numbers $i_{1}, \ldots, i_{m}$ are not pairwise distinct.

We say that $D$ is realized by a tree $T$ if the leaf set of $T$ is $[n]$ and if for each $m$-subset $V=\left\{i_{1}, \ldots, i_{m}\right\} \subset[n]$, the weight of the smallest subtree of $T$ containing $V$ is equal to $D\left(i_{1}, \ldots, i_{m}\right)$. An important result on $m$-dissimilarity maps of trees is given in [5].

Theorem 1.3. Let $T$ be a tree with $n$ leaves and no vertices of degree 2. Let $m \geq 3$ be an integer. If $n \geq 2 m-1$, then $T$ is uniquely determined by its $m$-dissimilarity map $D$. If $n=2 m-2$, this is not true.

In this paper, we give a description of a map $\phi^{(m)}: \mathbb{R}^{\left(\begin{array}{c}n \\ 2\end{array}\right)} \rightarrow \mathbb{R}^{\left(\begin{array}{c}n \\ 3\end{array}\right) \text {, sending }}$ the distance matrix of a tree $T$ to its corresponding $m$-dissimilarity map (see Theorem 3.2 in Section (3). In Section 4, we investigate the case $m=3$. In particular, we show that $\phi^{(3)}\left(\mathcal{T}_{n}\right)$ is equal to the intersection of the tropical Grassmannian $\mathcal{G}_{3, n}$ with a linear space (see Theorem 4.6). In Section 5 , we give a partial result on the case $m=4$. An introduction to tropical gemetry is given in Section 2 ,

To finish this section, we describe the relation with Phylogenetics. A classical problem in computational biology is to construct a phylogenetic tree from a sequence alignment of $n$ species

\begin{tabular}{|c|c|}
\hline Species 1 & ACAATGTCATTAGCGATACGTAGGTACGATGC. \\
\hline Species 2 & ACGTTGTCAATAGAGATTTTGGATGAACGATA. \\
\hline Species 3 & ACGTAGTCATTACACATTCTGGATTAACGTTA. \\
\hline Species 4 & GCACAGTCAGTAGAAGCTATGGTACATCGATC. \\
\hline & $\vdots$ \\
\hline
\end{tabular}

The main technique to select a tree model is computing the maximum likelihood estimate (MLE) for each of the $(2 n-5) !$ ! trees. Unluckily, all the MLE 
computations are very difficult, even for a single tree, and this approach requires examining all exponentially many trees.

A popular way to avoid this problem is the so-called distance based approach, where one collapses the data to a dissimilarity matrix and obtains a tree via a projection onto tree space $\mathcal{T}_{n}$ (by using the neighbor-joining algorithm). In fact, for such sequence data, computational biologists infer the distance between any two taxa. Thus, an interesting problem of phylogenetics concerns the construction of a weighted tree which represents this distance matrix, provided such a tree exists.

More general, we may think of an $m$-dissimilarity map as a measure of how dissimilar each subset of $m$ species is. As a generalization of the previous problem, we can search for a weighted tree such that the $m$-subtree weights represent the entries of the $m$-dissimilarity map. This problem has some natural relevance in Phylogenetics. Indeed, for example, it can be more reliable statistically to estimate the triple weights $D(i, j, k)$ rather than the pairwise distances $D(i, j)$ ([5], [6]).

\section{Tropical geometry}

The tropical semiring $(\mathbb{R} \cup\{-\infty\}, \oplus, \otimes)$ is the set of real numbers completed with $-\infty$, equiped with two binary operations: the tropical sum is the maximum of two numbers and the tropical multiplication is the ordinary sum.

Tropical monomials $x_{1}^{a_{1}} \cdots x_{k}^{a_{k}}$ represent ordinary linear forms $\sum_{i=1}^{k} a_{i} x_{i}$ and tropical polynomials

$$
\bigoplus_{a \in A} \lambda_{a} \otimes x_{1}^{a_{1}} \otimes \cdots \otimes x_{k}^{a_{k}}
$$

with $A \subset \mathbb{N}^{k}$ finite and $\lambda_{a} \in \mathbb{R}$, represent piecewise-linear convex functions

$$
F: \mathbb{R}^{k} \rightarrow \mathbb{R}:\left(x_{1}, \ldots, x_{k}\right) \mapsto \max _{a \in A}\left\{\lambda_{a}+\sum_{i=1}^{k} a_{i} x_{i}\right\} .
$$

Now let $K$ be the field of Puiseux series, i.e. the field of formal power series $a=\sum_{q \in \mathbb{Q}} a_{q} t^{q}$ in the variable $t$ such that the set $Q_{a}=\left\{q \in \mathbb{Q} \mid a_{q} \neq 0\right\}$ is bounded below and has a finte set of denominators. For such an $a$, the infimum of $Q_{a}$ is equal to the minimum and we call it the valuation $\operatorname{val}(a)$ of $a$.

A polynomial

$$
f\left(x_{1}, \cdots, x_{k}\right)=\sum_{a \in A} g_{a}(t) x_{1}^{a_{1}} \cdots x_{k}^{a_{k}} \in K[X]
$$

gives rise to the tropical polynomial in (1), where $\lambda_{a}=-\operatorname{val}\left(g_{a}(t)\right)$. We denote this tropical polynomial by $\operatorname{trop}(f)$.

We define the tropical hypersurface $\mathcal{T}(F)=\mathcal{T}(\operatorname{trop}(f))$ as the corner locus of the function $F$ in (2), i.e. the set of $x=\left(x_{1}, \ldots, x_{k}\right) \in \mathbb{R}^{k}$ such that the 
maximum of the collection of numbers

$$
\left\{\sum_{i=1}^{k} a_{i} x_{i}+\lambda_{a}\right\}_{a \in \mathcal{A}}
$$

is attained at least twice.

Theorem 2.1. If $I \subset K\left[x_{1}, \ldots, x_{n}\right]$ is an ideal, the following two subsets of $\mathbb{R}^{k}$ coincide:

1. the intersection of all tropical hypersurfaces $\mathcal{T}(\operatorname{trop}(f))$ with $f \in I$;

2. the closure in $\mathbb{R}^{k}$ of the set

$$
\left\{\left(-\operatorname{val}\left(y_{1}\right), \ldots,-\operatorname{val}\left(y_{k}\right)\right) \mid\left(y_{1}, \ldots, y_{k}\right) \in V(I)\right\} \subset \mathbb{Q}^{k} .
$$

Proof. See [7, Theorem 2.1].

For an ideal $I \subset K\left[x_{1}, \ldots, x_{k}\right]$, we denote by $\mathcal{T}(I) \subset \mathbb{R}^{k}$ the set mentioned in Theorem 2.1, It is called the tropical variety of the ideal $I$.

Definition 2.2. If $\mathcal{T}(I) \subset \mathbb{R}^{k}$ is a tropical variety, we say that $\left\{f_{1}, \ldots, f_{r}\right\}$ is a tropical basis of $\mathcal{T}(I)$ if and only if $I=\left\langle f_{1}, \ldots, f_{r}\right\rangle$ and

$$
\mathcal{T}(I)=\mathcal{T}\left(\operatorname{trop}\left(f_{1}\right)\right) \cap \cdots \cap \mathcal{T}\left(\operatorname{trop}\left(f_{r}\right)\right) .
$$

Remark 2.3. In general, a set of generators of an ideal $I$ is not a tropical basis for $\mathcal{T}(I)$. Of course, the singleton $\{f\}$ is a tropical basis for the tropical hypersurface $\mathcal{T}(\operatorname{trop}(f))$.

We are mainly interested in the tropical variety $\mathcal{T}\left(I_{m, n}\right)$, where $I_{m, n}$ is the ideal of the Grassmannian $G(m, n) \subset \mathbb{R}^{\left(\begin{array}{c}n \\ m\end{array}\right)}$. To be more precise, we fix a polynomial ring

$$
\mathbb{Z}[x]=\mathbb{Z}\left[x_{i_{1} i_{2} \cdots i_{d}} \mid 1 \leq i_{1}<i_{2}<\cdots<i_{m} \leq n\right]
$$

in $\left(\begin{array}{c}n \\ m\end{array}\right)$ variables with integer coefficients. The Plücker ideal $I_{m, n}$ is the prime ideal in $\mathbb{Z}[x]$, consisting of the algebraic relations among the determinants of the $(m \times m)$-minors of any $(m \times n)$-matrix with entries in a commutative ring. It is well-known that $I_{m, n}$ is generated by quadrics (see for example [8]).

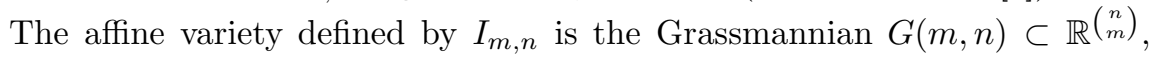
which parameterizes all $m$-dimensional linear subspaces of an $n$-dimensional vector space. It has dimension $(n-m) m+1$.

Definition 2.4. The tropical variety $\mathcal{T}\left(I_{m, n}\right)$ is called a tropical Grassmannian and is denoted by $\mathcal{G}_{m, n}$.

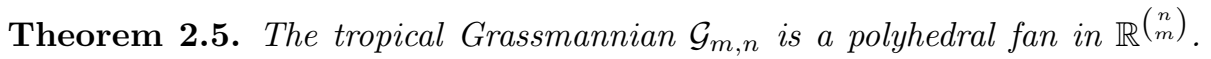
Each of its maximal cones has the same dimension, namely $(n-m) m+1$. 
Proof. See [7, Corollary 3.1.].

Now we are going to fix our attention on the case $m=2$.

Example 2.6 $(m=2$ and $n=4)$. The smallest non-zero Plücker ideal is the principal ideal $I_{2,4}=\left(x_{12} x_{34}-x_{13} x_{24}+x_{14} x_{23}\right)$. Thus $\mathcal{G}_{2,4}$ is a fan with three five-dimensional cones $\mathbb{R}^{4} \times \mathbb{R}_{\leq 0}$ glued along $\mathbb{R}^{4}$.

Theorem 2.7. The ideal $I_{2, n}$ is generated by the quadratic polynomials

$$
p_{i j k l}:=\underline{x_{i k} x_{j l}}-x_{i j} x_{k l}-x_{i l} x_{j k} \quad(1 \leq i<j<k<l \leq n) .
$$

These polynomials form the reduced Gröbner basis if the underlined terms are leading.

Proof. See [8, Theorem 3.1.7 and Proposition 3.7.4].

For each quadruple $\{i, j, k, l\} \subset\{1,2, \ldots, n\}$, we consider the tropical polynomial

$$
\operatorname{trop}\left(p_{i j k l}\right)=\left(x_{i j} \otimes x_{k l}\right) \oplus\left(x_{i k} \otimes x_{j l}\right) \oplus\left(x_{i l} \otimes x_{j k}\right) .
$$

This polynomial defines a tropical hypersurface $\mathcal{T}\left(\operatorname{trop}\left(p_{i j k l}\right)\right)$. It turns out that the tropical Grassmannian $\mathcal{G}_{2, n}$ is the intersection of these $\left(\begin{array}{l}n \\ 4\end{array}\right)$ hypersurfaces, so the quadrics $p_{i j k l}$ forms a tropical basis for $I_{2, n}$ (see [7]).

Let $D$ be an dissimilarity matrix on $[n]$ and $\{i, j, k, l\} \subset[n]$. The maximum of the three numbers $D(i, j)+D(k, l), D(i, k)+D(j, l)$ and $D(i, l)+D(j, k)$ is attained at least twice if and only if $D \in \mathcal{T}\left(\operatorname{trop}\left(p_{i j k l}\right)\right)$. Thus Theorem 1.1 implies that a metric $D$ on $[n]$ is a tree metric if and only if $D$ belongs to $\mathcal{T}_{n}$. In particular, one has the following result.

Theorem 2.8. The space of trees $\mathcal{T}_{n}$ is the tropical Grassmannian $\mathcal{G}_{2, n}$.

Proof. See [7, Theorem 4.2] or the arguments above.

Now we come back to the general case (so the case where $m \leq n$ is arbitrary). The ideal $I_{m, n}$ is generated by quadratic polynomials, known as the Plücker relations. Among these are the three-term Plücker relations

$$
p_{R, i j k l}:=x_{R i k} x_{R j l}-x_{R i j} x_{R k l}-x_{R i l} x_{R j k},
$$

which are closely related to (3). Hereby $R$ is any $(m-2)$-subset of $[n]$ and $i, j, k, l \in[n] \backslash R$.

Definition 2.9. The three-term tropical Grassmannian $\mathcal{T}_{m, n}$ is the intersection

$$
\mathcal{T}_{m, n}:=\bigcap_{R, i, j, k, l} \mathcal{T}\left(\operatorname{trop}\left(p_{R, i j k l}\right)\right) \subset \mathbb{R}^{\left(\begin{array}{l}
n \\
m
\end{array}\right)} .
$$

In general, the three-term Plücker relations do not generate $I_{m, n}$. If $m=2$, then $S=\emptyset$ and $\mathcal{T}_{2, n}=\mathcal{G}_{2, n}$. For $m \geq 3$, the tropical Grassmannian $\mathcal{G}_{m, n}$ is contained in $\mathcal{T}_{m, n}$. This containment is proper for $n \geq m+4$. 


\section{A description on the $m$-subtree weight map}

In this section, we are going to give an explicit description of a map

$$
\phi^{(m)}: \mathbb{R}^{\left(\begin{array}{l}
n \\
2
\end{array}\right)} \rightarrow \mathbb{R}^{\left(\begin{array}{c}
n \\
m
\end{array}\right)},
$$

sending the dissimilarity matrix $D$ of a tree $T$ to its $m$-dissimilarity map.

Let $\prec$ be the order relation on $\mathbb{N}^{\infty}$ defined as follows. We have

$$
\left(a_{1}, a_{2}, a_{3}, \ldots\right) \prec\left(b_{1}, b_{2}, b_{3}, \ldots\right)
$$

if and only if there exists an $n \in \mathbb{N}$ such that $a_{i}=b_{i}$ for all $i<n$ and $a_{n}<b_{n}$.

Let $T$ be a tree with $n$ leaves. Let $r$ be an inner node of $T$ and consider $T$ as a rooted tree (with root $r$ ). Let $\mathcal{N}$ be the set of nodes of $T$. In particular, the set of leaves $[n]=\{1, \ldots, n\}$ is contained in $\mathcal{N}$.

Lemma 3.1. There exists a map $\alpha: \mathcal{N} \rightarrow \mathbb{N}^{\infty}$ such that the following properties hold:

1. $\alpha$ is injective.

2. If $n \in \mathcal{N}$ is an ancestor of $m \in \mathcal{N}$, we have $\alpha(m) \succ \alpha(n)$. So the root $r$ of $T$ gives rise to the minimum of $\{\alpha(n) \mid n \in \mathcal{N}\}$.

3. If $n_{1}, n_{2} \in \mathcal{N}$ with $n_{2}$ not a descendant nor an ancestor of $n_{1}, m_{1} \in \mathcal{N} a$ descendant of $n_{1}$ and $m_{2} \in \mathcal{N}$ a descendant of $n_{2}$, we have $\alpha\left(m_{1}\right) \prec \alpha\left(m_{2}\right)$ if and only if $\alpha\left(n_{1}\right) \prec \alpha\left(n_{2}\right)$.

Proof. We will define $\alpha$ inductively. Take $\alpha(r)=(0,0,0, \ldots)$. For the induction step, if $\alpha(n)=\left(a_{1}, \ldots, a_{s}, 0,0, \ldots\right)$ is defined for some $n \in \mathcal{N}$ with $a_{s} \neq 0$ and if $m_{1}, \ldots, m_{t}$ are the children of $n$, take $\alpha\left(n_{i}\right)=\left(a_{1}, \ldots, a_{s}, i, 0, \ldots\right)$. Note that all the properties hold and that the depth of $n \in \mathcal{N}$ in $T$ is equal to the number of non-zero entries in $\alpha(n)$.

We say that the leaves of $T$ are well-numbered if and only if $\alpha(i) \prec \alpha(j)$ for all $i<j$.

A permutation $\sigma \in S_{m}$ of $\{1, \ldots, m\}$ is called cyclic if and only if the decomposition of $\sigma$ into a product of disjoint cycles consists of only one cycle of order $m$. Denote the set of cyclic permutations in $\mathcal{S}_{m}$ by $\mathcal{C}_{m}$. Note that $\sigma^{m}=I d$ if $\sigma \in \mathcal{C}_{m}$

Theorem 3.2. Let $n$ and $m$ be integers such that $n>m \geq 2$. Let

$$
\phi^{(m)}: \mathbb{R}^{\left(\begin{array}{c}
n \\
2
\end{array}\right)} \rightarrow \mathbb{R}^{\left(\begin{array}{c}
n \\
m
\end{array}\right)}: X=\left(X_{i, j}\right) \mapsto\left(X_{i_{1}, \ldots, i_{m}}\right)
$$

be the map with

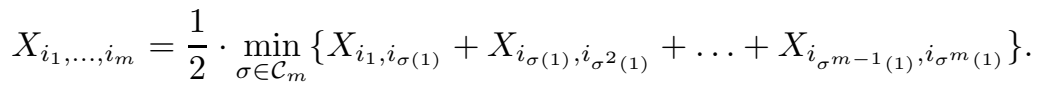

If $D \in \mathcal{G}_{2, n} \subset \mathbb{R}^{\left(\begin{array}{l}n \\ 2\end{array}\right)}$ is the dissimilarity matrix of an $n$-tree $T$, then the $m$ dissimilarity map of $T$ is equal to $\phi^{(m)}(D)$. So the set of $m$-dissimilarity maps of $n$-trees is equal to $\phi^{(m)}\left(\mathcal{G}_{2, n}\right)$. 
Proof. Write

$$
f\left(X ; \sigma ; i_{1}, \ldots, i_{m}\right)=X_{i_{1}, i_{\sigma(1)}}+X_{i_{\sigma(1)}, i_{\sigma^{2}(1)}}+\ldots+X_{i_{\sigma^{m-1}(1)}, i_{\sigma m}(1)} .
$$

Note that

$$
f\left(X ; \sigma ; i_{\pi(1)}, \ldots, i_{\pi(m)}\right)=f\left(X ; \pi \sigma \pi^{-1} ; i_{1}, \ldots, i_{m}\right)
$$

for all $\pi \in \mathcal{S}_{m}$, hence

$$
\min _{\sigma \in \mathcal{C}_{m}}\left\{f\left(X ; \sigma ; i_{\pi(1)}, \ldots, i_{\pi(m)}\right)\right\}=\min _{\sigma \in \mathcal{C}_{m}}\left\{f\left(X ; \sigma ; i_{1}, \ldots, i_{m}\right)\right\} .
$$

We have to prove that the weight $D\left(i_{1}, \ldots, i_{m}\right)$ of the smallest subtree $T^{\prime}$ of $T$ containing the leaves $i_{1}, \ldots, i_{m}$ is equal to $\frac{1}{2} \cdot \min _{\sigma \in \mathcal{C}_{m}}\left\{f\left(D ; \sigma ; i_{1}, \ldots, i_{m}\right)\right\}$. It is enough to prove this for $i_{1}=1, \ldots, i_{m}=m$ (the general case is proved completely analogously). By equation (4), we may also assume the leaves of $T^{\prime}$ are well-numbered.

Let $e=(x, y)$ be an edge of $T^{\prime}$ with $y$ a child of $x$. We claim that for all $\sigma \in \mathcal{C}_{m}$, the weight $w(e)$ of $e$ is taken into account in at least two of the $m$ terms of

$$
f(D ; \sigma ; 1, \ldots, m)=D(1, \sigma(1))+D\left(\sigma(1), \sigma^{2}(1)\right)+\ldots+D\left(\sigma^{m-1}(1), 1\right)
$$

and in exactly two of the summands of

$$
f(D ; \tau ; 1, \ldots, m)=D(1,2)+D(2,3)+\ldots+D(m, 1),
$$

where

$$
\tau=\left(\begin{array}{ccccc}
1 & 2 & \ldots & m-1 & m \\
2 & 3 & \ldots & m & 1
\end{array}\right) \in \mathcal{C}_{m}
$$

Using this claim, we immediately see

$$
D\left(i_{1}, \ldots, i_{m}\right)=\frac{1}{2} \cdot f(D ; \tau ; 1, \ldots, m)=\frac{1}{2} \cdot \min _{\sigma \in \mathcal{C}_{m}}\{f(D ; \sigma ; 1, \ldots, m)\} .
$$

To finish this theorem, we only need to prove the claim. Consider the split of $T^{\prime}$ induced by $e$ and let $T^{\prime \prime}$ be the component of the split containing $y$ (hence $T^{\prime \prime}$ is the maximal subtree of $T^{\prime}$ containing $y$ but not $x$ ). Denote the set of leaves of $T^{\prime \prime}$ by $L^{\prime \prime}$. We may assume $1 \in L^{\prime \prime}$ (the case $1 \notin L^{\prime \prime}$ is analogous). Note that in this case $L^{\prime \prime}$ is of the form $\{1, \ldots, s\}$ for some $s<m$.

The weight of $e$ is taken into account in the term $D(i, j)$ (i.e. the path between the leaves $i$ and $j$ of $T^{\prime \prime}$ passes $e$ ) if and only if $i \in L^{\prime \prime}$ and $j \notin L^{\prime \prime}$ or vice versa. Thus $w(e)$ is only counted in the two terms $D(s, s+1)$ and $D(m, 1)$ of $f(D ; \tau ; 1, \ldots, m)$.

So it is enough to show that there exists a $t \in\{0 \ldots, m-1\}$ such that $\sigma^{t}(1) \in$ $L^{\prime \prime}$ and $\sigma^{t+1}(1) \notin L^{\prime \prime}$ (the other case is proved analogously). If we assume this is not the case (so $\sigma^{t}(1) \in L^{\prime \prime}$ implies $\sigma^{t+1}(1) \in L^{\prime \prime}$ ), we get $L^{\prime \prime}=\{1, \ldots, m\}$, a contradiction. 


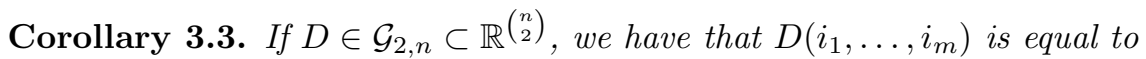

$$
\left(\bigoplus_{\sigma \in \mathcal{C}_{m}}\left(D\left(i_{1}, i_{\sigma(1)}\right) \otimes D\left(i_{\sigma(1)}, i_{\sigma^{2}(1)}\right) \otimes \cdots \otimes D\left(i_{\sigma^{m-1}(1)}, i_{\sigma^{m}(1)}\right)\right)^{-1}\right)^{-\frac{1}{2}} .
$$

Remark 3.4. In each component $D\left(i_{1}, \ldots, i_{m}\right)$, the minimum is attained at least twice. Indeed, assume the minimum is attained for $\sigma=\tau$. Since

$$
f\left(D ; \tau ; i_{1}, \ldots, i_{m}\right)=f\left(D ; \tau^{-1} ; i_{1}, \ldots, i_{m}\right),
$$

the minimum is also attained for $\sigma=\tau^{-1}$. Note that this could be useful for computations, since it permits us to consider only $\frac{\left|\mathcal{C}_{m}\right|}{2}$ permutations. Furthermore, if $\left\{i_{j}, i_{k}\right\}$ is a cherry of $T^{\prime}$, the minimum is also attained for $\sigma=(j k) \circ \tau \circ(j k)$, whereby $(j k)$ is the transposition in $\mathcal{S}_{m}$ switching $j$ and $k$.

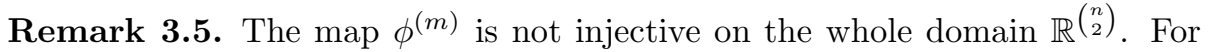

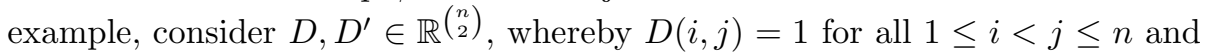
$D^{\prime}$ only differs from $D$ in the last coordinates, with $D^{\prime}(n-1, n)=2$. Clearly, one has $D \in \mathcal{G}_{2, n}, D^{\prime} \notin \mathcal{G}_{2, n}$ and $\phi^{(m)}(D)=\phi^{(m)}\left(D^{\prime}\right)$. However, Theorem 1.3 implies that the restriction of $\phi^{(m)}$ to $\mathcal{G}_{2, n}$ is injective if $n \geq 2 m-1$.

Proposition 3.6. $\phi^{(m)}\left(\mathcal{G}_{2, n}\right) \subseteq \mathcal{T}_{m, n} \cap \phi^{(m)}\left(\mathbb{R}^{\left(\begin{array}{l}n \\ 2\end{array}\right)}\right)$

Proof. The inclusion $\phi^{(m)}\left(\mathcal{G}_{2, n}\right) \subset \phi^{(m)}\left(\mathbb{R}^{\left.\left(\begin{array}{l}n \\ 2\end{array}\right)\right)}\right)$ is obvious, while $\phi^{(m)}\left(\mathcal{G}_{2, n}\right) \subset$ $\mathcal{T}_{m, n}$ follows from [5]. For sake of completeness, we include the proof in this paper.

Consider a tree $T$ with leaf set $[n]$ and distance matrix $D$. Let $R$ be an $(m-2)$-subset of $[n]$ and $i, j, k, l \in[n] \backslash R$. We have to prove that

$$
\phi^{(m)}(D) \in \mathcal{T}\left(\operatorname{trop}\left(p_{R, i j k l}\right)\right) .
$$

Let $[R]$ be the smallest subtree of $T$ containing the leaves in $R$ and let $T^{\prime}$ be the tree obtained from $T$ by contracting $[R]$ to a point. Denote by $i^{\prime}, j^{\prime}$, etc. the images of respectively $i, j$, etc. in $T^{\prime}$. Note that $R^{\prime}$ is a leaf of $T^{\prime}$. We have

$$
D(R, i, j)=D^{\prime}\left(R^{\prime}, i^{\prime}, j^{\prime}\right)+D(R),
$$

hence $\phi^{(m)}(D) \in \mathcal{T}\left(\operatorname{trop}\left(p_{R, i j k l}\right)\right)$ if and only if $\phi^{(3)}\left(D^{\prime}\right) \in \mathcal{T}\left(\operatorname{trop}\left(p_{R^{\prime}, i^{\prime} j^{\prime} k^{\prime} l^{\prime}}\right)\right)$, where $D^{\prime}$ is the distance matrix of $T^{\prime}$.

Now Remark 4.1 below implies

$$
D^{\prime}\left(R^{\prime}, i^{\prime}, j^{\prime}\right)=\frac{1}{2}\left(D^{\prime}\left(i^{\prime}, j^{\prime}\right)+D^{\prime}\left(i^{\prime}, R^{\prime}\right)+D^{\prime}\left(j^{\prime}, R^{\prime}\right)\right),
$$

so $\phi^{(3)}\left(D^{\prime}\right) \in \mathcal{T}\left(\operatorname{trop}\left(p_{R^{\prime}, i^{\prime} j^{\prime} k^{\prime} l^{\prime}}\right)\right)$ if and only if $D^{\prime} \in \mathcal{T}\left(\operatorname{trop}\left(p_{i^{\prime} j^{\prime} k^{\prime} l^{\prime}}\right)\right)$. Hence the statement follows from Theorem 1.1 . 


\section{The 3-dissimilarity maps of trees}

Denote the coordinates of $\mathbb{R}^{\left(\begin{array}{c}n \\ 2\end{array}\right)}$ by $X(i, j)$ (here we index over all integers $i, j$ with $1 \leq i<j \leq n$ ) and the coordinates of $\mathbb{R}^{\left(\begin{array}{l}n \\ 3\end{array}\right)}$ by $X(i, j, k)$ (here we index over all integers $i, j, k$ with $1 \leq i<j<k \leq n$ ). Recall that if $D \in \mathcal{G}_{2, n}$ is a tree, $D(i, j)$ is the distance between leaf $\mathrm{i}$ and leaf $\mathrm{j}$.

Remark 4.1. Since $\mathcal{C}_{3}=\left\{\sigma_{1}, \sigma_{2}\right\}$ with

$$
\sigma_{1}=\left(\begin{array}{lll}
1 & 2 & 3 \\
2 & 3 & 1
\end{array}\right) \quad \text { and } \quad \sigma_{2}=\left(\sigma_{1}\right)^{-1}=\left(\begin{array}{lll}
1 & 2 & 3 \\
3 & 1 & 2
\end{array}\right)
$$

the $\operatorname{map} \phi^{(3)}$ sends $X=(X(i, j))_{i, j}$ to $(X(i, j, k))_{i, j, k}$ with

$$
X(i, j, k)=\frac{1}{2} \cdot(X(i, j)+X(i, k)+X(j, k)) .
$$

So if $D \in \mathcal{G}_{2, n}$, the 3 -subtree weights of the tree $D$ are given by $D(i, j, k)=$ $\frac{1}{2} \cdot(D(i, j)+D(i, k)+D(j, k))$.

The following results states that for the case $m=3$ the equality holds in Proposition 3.6 if $n \geq 5$.

Proposition 4.2. If $n \geq 5$, we have $\phi^{(3)}\left(\mathcal{G}_{2, n}\right)=\mathcal{T}_{3, n} \cap \phi^{(3)}\left(\mathbb{R}_{\left(\begin{array}{l}n \\ 2\end{array}\right)}\right)$

Proof. By Proposition 3.6, it is enough to show that for a general point $P \in$ $\phi^{(3)}\left(\mathbb{R}^{\left(\begin{array}{l}n \\ 2\end{array}\right)}\right) \cap \mathcal{T}_{3, n}$, there exists a point $D \in \mathcal{G}_{2, n}$ such that $\phi^{(3)}(D)=P$. Since $P \in \phi^{(3)}\left(\mathbb{R}^{\left(\begin{array}{l}n \\ 2\end{array}\right)}\right)$, there exists a point $D \in \mathbb{R}^{\left(\begin{array}{l}n \\ 2\end{array}\right)}$ such that $\phi^{(3)}(D)=P$. It suffices to prove that $D \in \mathcal{G}_{2, n}$. In order to do this, we show that in each triplet

$$
\{D(i, j)+D(k, l), D(i, k)+D(j, l), D(i, k)+D(j, k)\},
$$

the maximum is attained at least twice. Fix $S \in[n] \backslash\{i, j, k, l\}(n \geq 5)$. Since $P \in \mathcal{T}_{3, n}$, in the triplet

$$
\{P(S, i, j)+P(S, k, l), P(S, i, k)+P(S, j, l), P(S, i, l)+P(S, j, k)\},
$$

the maximum is attained at least twice. Note that

$$
\begin{aligned}
& P(S, i, j)+P(S, k, l)=\frac{1}{2}(C+D(i, j)+D(k, l)), \\
& P(S, i, k)+P(S, j, l)=\frac{1}{2}(C+D(i, k)+D(j, l)), \\
& P(S, i, l)+P(S, j, k)=\frac{1}{2}(C+D(i, k)+D(j, k)),
\end{aligned}
$$

where $C=D(S, i)+D(S, j)+D(S, k)+D(S, l)$. Hence the maximum in $\{D(i, j)+D(k, l), D(i, k)+D(j, l), D(i, k)+D(j, k)\}$ is also attained at least twice, thus $D \in \mathcal{G}_{2, n}$ and $P \in \phi^{(3)}\left(\mathcal{G}_{2, n}\right)$. 
For the proof of the proposition below, we need an extra definition.

Definition 4.3. An ultrametric $D$ on $[n]$ is a metric which satisfies the following strengthened version of the triangle inequality:

$$
\forall i, j, k \in[n]: D(i, j) \leq \max \{D(i, k), D(j, k)\} .
$$

Equivalently, at least two of the three terms $D(i, j), D(i, k), D(j, k)$ are the same.

Remark 4.4. In general, the dissimilarity matrix $D$ of a tree $T$ is not an ultrametric. In case $D \in \mathcal{G}_{2, n}$ is an ultrametric, we can realize $D$ by an equidistant tree, i.e. a rooted tree such that the distance $F$ between the root and each leaf is equal. In particular, $2 F=\max \{D(i, j) \mid i, j \in X$ and $i \neq j\}$.

Proposition 4.5. $\phi^{(3)}\left(\mathcal{G}_{2, n}\right) \subset \mathcal{G}_{3, n}$

Proof. Let $T$ be a tree with 3-dissimilarity map

$$
P=(D(i, j, k))_{i, j, k}=\phi^{(3)}\left(\left(D(i, j)_{i, j}\right) \in \phi^{(3)}\left(\mathcal{G}_{2, n}\right) \subset \mathbb{R}^{\left(\begin{array}{c}
n \\
3
\end{array}\right)} .\right.
$$

If $M \in K^{3 \times n}$, we denote the $(3 \times 3)$-minor with columns $i, j, k$ by $M(i, j, k)$. By Theorem 2.1, $\mathcal{G}_{3, n}$ is the closure in $\mathbb{R}^{\left(\begin{array}{l}n \\ 3\end{array}\right)}$ of the set

$$
S:=\left\{(-\operatorname{val}(\operatorname{det}(M(i, j, k))))_{i, j, k} \mid M \in K^{3 \times n}\right\} \subset \mathbb{Q}^{\left(\begin{array}{c}
n \\
3
\end{array}\right) .} .
$$

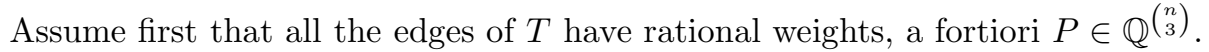
We are going to show there exists a matrix $M \in K^{3 \times n}$ such that

$$
D(i, j, k)=-\operatorname{val}(\operatorname{det}(M(i, j, k)))
$$

Fix a rational number $E$ with $E \geq D(i, n)$ for all $i \in\{1, \ldots, n-1\}$ and define a new metric $D^{\prime}$ by

$$
D^{\prime}(i, j)=2 E+D(i, j)-D(i, n)-D(j, n)
$$

for all different $i, j \in[n]$ (in particular, $D^{\prime}(i, n)=2 E$ for $i \neq n$ ). Note that $D^{\prime} \in \mathcal{G}_{2, n}$ and that $D^{\prime}$ an ultrametric on $\{1, \ldots, n-1\}$, so it can be realized by an equidistant $(n-1)$-tree $T^{\prime \prime}$ with root $r$. Each edge $e$ of $T^{\prime \prime}$ has a well-defined height $h(e)$, which is the distance from the top node of $e$ to each leaf below $e$. Pick a random rational number $a(e)$ and associate the label $a(e) t^{2 h(e)}$ to $e$. If $i \in\{1, \ldots, n-1\}$ is a leaf of $T^{\prime \prime}$, define the polynomial $x_{i}(t)$ by adding the labels of all edges between $r$ and $i$. It is easy to see that $D^{\prime}(i, j)=\operatorname{deg}\left(x_{j}(t)-x_{i}(t)\right)$ for all $i, j \in\{1, \ldots, n-1\}$.

Denote the distance from $r$ to each edge by $F$. Since

$$
2 F=\max \left\{D^{\prime}(i, j) \mid 1 \leq i<j \leq n-1\right\}<2 E,
$$

we have $F<E$. The metric $D^{\prime}$ on $[n]$ can be realized by a tree $T^{\prime}$, where $T^{\prime}$ is the tree obtained from $T^{\prime \prime}$ by adding the leave $n$ together with an edge $(r, n)$ of 
length $2 E-F$. If we define $x_{n}(t)=t^{2 E}$, we get that $D^{\prime}(i, j)=\operatorname{deg}\left(x_{j}(t)-x_{i}(t)\right)$ for all $i, j \in[n]$.

Now consider the matrix

$$
M^{\prime}=\left[\begin{array}{ccccc}
1 & 1 & 1 & \ldots & 1 \\
x_{1}(t) & x_{2}(t) & x_{3}(t) & \ldots & x_{n}(t) \\
x_{1}(t)^{2} & x_{2}(t)^{2} & x_{3}(t)^{2} & \ldots & x_{n}(t)^{2}
\end{array}\right] .
$$

We have $\operatorname{det}\left(M^{\prime}(i, j, k)\right)=\left(x_{j}(t)-x_{i}(t)\right)\left(x_{k}(t)-x_{i}(t)\right)\left(x_{k}(t)-x_{j}(t)\right)$, hence

$$
D^{\prime}(i, j)+D^{\prime}(i, k)+D^{\prime}(j, k)=\operatorname{deg}\left(\operatorname{det}\left(M^{\prime}(i, j, k)\right)\right) .
$$

Let $M$ be the matrix obtained from $M^{\prime}$ by multiplying, for each $i$, the $i$-th column of $M^{\prime}$ by $\left(t^{D(i, n)-E}\right)^{2}$. Since

$$
\begin{aligned}
D(i, j) & =D^{\prime}(i, j)+(D(i, n)-E)+(D(j, n)-E) \\
& =\operatorname{deg}\left(t^{D(i, n)-E} \cdot t^{D(j, n)-E} \cdot\left(x_{i}(t)-x_{j}(t)\right)\right),
\end{aligned}
$$

we get that $D(i, j)+D(i, k)+D(j, k)=\operatorname{deg}(\operatorname{det}(M(i, j, k)))$. If we replace each $t$ in $M$ by $t^{-1 / 2}$, we get

$$
D(i, j, k)=-\operatorname{val}(\operatorname{det}(M(i, j, k))) .
$$

Now assume $T$ has irrational edge weights. We can approximate $T$ arbitrarily close by a tree $\widetilde{T}$ with rational edge weights. From the arguments above, it follows that the 3 -dissimilarity map $\widetilde{D}$ of $\widetilde{T}$ belongs to $S$, hence $D \in \mathcal{G}_{3, n}$.

Theorem 4.6. If $n \geq 5$, we have $\phi^{(3)}\left(\mathcal{G}_{2, n}\right)=\phi^{(3)}\left(\mathbb{R}^{\left(\begin{array}{l}n \\ 3\end{array}\right)}\right) \cap \mathcal{G}_{3, n}$.

Proof. The statement follows from Proposition 4.2, Proposition4.5 and the fact that $\mathcal{G}_{3, n} \subset \mathcal{T}_{3, n}$.

\section{The 4-dissimilarity maps of trees}

In this section, we give a geometric description of $\phi^{(4)}\left(\mathcal{G}_{2, n}\right)$.

Remark 5.1. The set $\mathcal{C}_{4}=\left\{\sigma_{1}, \sigma_{1}^{-1}, \sigma_{2}, \sigma_{2}^{-1}, \sigma_{3}, \sigma_{3}^{-1}\right\}$ with

$$
\sigma_{1}=\left(\begin{array}{cccc}
1 & 2 & 3 & 4 \\
2 & 3 & 4 & 1
\end{array}\right), \sigma_{2}=\left(\begin{array}{cccc}
1 & 2 & 3 & 4 \\
2 & 4 & 1 & 3
\end{array}\right), \sigma_{3}=\left(\begin{array}{cccc}
1 & 2 & 3 & 4 \\
3 & 4 & 2 & 1
\end{array}\right)
$$

Hence the map $\phi^{(4)}$ sends $(X(i, j))_{i, j}$ to $(X(i, j, k, l))_{i, j, k, l}$ where $X(i, j, k, l)$ is equal to the minimum of the three terms

$$
\begin{aligned}
& X(1,2)+X(2,3)+X(3,4)+X(4,1), \\
& X(1,2)+X(2,4)+X(4,3)+X(3,1), \\
& X(1,3)+X(3,2)+X(2,4)+X(4,1),
\end{aligned}
$$

divided by two. 


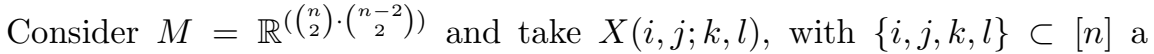
quadruple, as coordinates on $M$. For example, $X(j, i ; l, k)=X(i, j ; k, l)$, but $X(i, k ; j, l) \neq X(i, j ; k, l)$ and $X(k, l ; i, j) \neq X(i, j ; k, l)$.

Let $\pi: \mathbb{R}^{\left(\begin{array}{l}n \\ 2\end{array}\right)} \rightarrow M:(X(i, j))_{i, j} \mapsto(X(i, j ; k, l))_{i, j, k, l}$ with

$X(i, j ; k, l)=\frac{1}{2} \cdot(X(i, j)+X(k, l)+\min \{X(i, k)+X(j, l), X(i, l)+X(j, k)\})$.

Let $L$ be the linear subspace of $M$ consisting of points $X(i, j ; k, l)$ with

$X(i, j ; k, l)=X(i, k ; j, l)=X(i, l ; j, k)=X(j, l ; i, k)=X(j, k ; i, l)=X(k, l ; i, j)$

for all different $i, j, k, l \in[n]$. Points in $L$ can be projected naturally to $\mathbb{R}^{\left(\begin{array}{l}n \\ 4\end{array}\right)}$ by sending $X(i, j ; k, l)$ to $X(i, j, k, l)$. Denote this projection by $p$.

Proposition 5.2. $\phi^{(4)}\left(\mathcal{G}_{2, n}\right)=p\left(\pi\left(\mathbb{R}^{\left(\begin{array}{l}n \\ 2\end{array}\right)}\right) \cap L\right)$.

Proof. Note that for any real numbers $a, b, c$, we have

$$
a+\min \{b, c\}=b+\min \{a, c\}=c+\min \{a, b\}
$$

if and only if $\max \{a, b, c\}$ is attained at least twice. If the latter holds, the terms in (51) are equal to $\min \{a+b, a+c, b+c\}$.

If we take $a=X(i, j)+X(k, l), b=X(i, k)+X(j, l)$ and $c=X(i, l)+X(j, k)$, the statement follows from the Tree Metric Theorem.

\section{Aknowledgments}

We thank Ruriko Yoshida, Anders Jensen and expecially Bernd Sturmfels for their many comments and suggestions which improved this manuscript. The second author is a postdoctoral fellow of the Research Foundation - Flanders (FWO).

\section{References}

[1] L. Billera, S. Holmes, K. Vogtman: Geometry of the space of phylogenetic trees, Advances in Applied Mathematics 27 (2001), 733-767.

[2] P. Buneman, A Note on the Metric Properties of Trees, J. Combinatorial Theory 17 (1974), 48-50.

[3] F. Chung, M. Garrett, R.L. Graham, D. Shalcross, Distance realization problems with applications to Internet tomography, J. Computer Systems and Sciences 63 (2001), 432-448.

[4] S.L. Hakimi, S.S. Yau, Distance matrix of a graph and its realizability, Quart. Appl. Math. 22 (1965), 305-317. 
[5] L. Pachter, D. Speyer, Reconstructing trees from subtree weights, Applied Mathematics Letters 17 (2004), 615-621.

[6] L. Pachter, B. Sturmfels, Algebraic statistics for computational biology, Cambridge University Press, New York 2005

[7] D. Speyer, B. Sturmfels, The Tropical Grassmannian, Adv. Geom. 4 (2004), 389-411.

[8] B. Sturmfels, Algorithms in Invariant Theory, Texts and Monographs in Symbolic Computation, Springer-Verlag, Vienna, 1993. 